\title{
Personalized Search Recommender System: State of Art, Experimental Results and Investigations
}

\author{
Janet Rajeswari ${ }^{\mathrm{a}}$, Shanmugasundaram Hariharan ${ }^{\mathrm{b}}$ \\ ${ }^{a}$ Janet Rajeswari, Research and Development Centre, Bharathiar University, Coimbatore- 641046, India \\ ${ }^{b}$ Department of Computer Science and Engineering, TRP Eningeering College, Trichy-62105, India
}

\begin{abstract}
Personalized recommender system has attracted wide range of attention among researchers in recent years. These recommender systems suggest products or services depending upon user's personal interest. There has been a huge demand for development of web search apps for gaining knowledge pertaining to user's choice. A strong knowledge base, type of approach for search and several other factors make it accountable for a good personalized web search engine. This paper presents the state of art, challenges and other issues in this context, thereby providing the need for an improved personalized system. The study carried out in this paper reports the overview of existing technologies for building a personalized recommender systems in social networking platforms. Study reported in this article seems to be promising and provides possibilities of research directions, pros \& cons and other alternatives.
\end{abstract}

Index Terms: Personalization, Search process, recommender system, review, Web 2.0, social networking, Ecommerce.

(C) 2016 Published by MECS Publisher. Selection and/or peer review under responsibility of the Research Association of Modern Education and Computer Science.

\section{Introduction}

Information extraction, knowledge sharing via social media has gained commercial interest among wide range of people, especially among young generation. In today's modern era, these social platforms are of greater importance depending on user's interest. Hence investigation on personalizing the web search process has gained huge attention. Currently almost all Web applications are not read only, but editable in nature.

\footnotetext{
* Corresponding author.

E-mail address:
} 


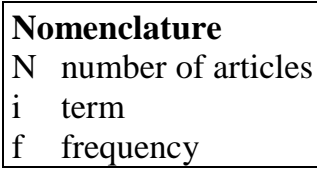

These social networks provide an environment to upload their personal information's like photographs, audio, videos, share bookmarks, write blogs, and annotating and commenting information provided by others. There are workgroups performing research on these Web 2.0 applications [2], since millions of users spend hours daily in these sites, generate rich information and share knowledge. The popularity of social networking sites results in flooding of information, overloading and several other complexities. A QoS aware recommender system [1] would a better solution.

Building a recommender system involves a strong background knowledge with ontology model [3], Support Vector Machine [4], ant colony optimization [5] to improve e-commerce [6]. Recommender systems field have been receiving growing attentions in data mining and among others in the recent years with quite a significant amount of research done in this area. The main aim of this study is to summarize the developments, pros and cons existing in several research strategies in recommender systems. Personalized recommender systems suggest products, services or reply catering the queries posted by the consumer that is proportional to the ecommerce growth. XING is the most powerful tool used for business social networking [7] which operates on 16 different languages. Recommender systems started its origin in mid 1990's using collaborative filtering approach involving user profiling. Profiling data includes users' selected items, ratings, data etc.

Traditionally recommender systems uses combinatory approaches involving user profiling, filtering and machine learning. In general, recommendation techniques are broadly classified as content-based filtering [8] and collaborative filtering [9]. Content-based filtering approaches are those which recommend items whose content is much more similar in content than previously viewed or selected. Collaborative filtering are those which collects information about a user by asking them to rate items and makes recommendations based on highly rated items. There are systems which combines both content and collaborative filtering approaches in order to make recommendations [10] using tag based approaches [26].

Recommender systems are broad classified based on the approach provided for recommendations based on the information filtering mechanism as content-based methods, collaborative methods and hybrid methods. Content-based (CB) recommenders provide recommendations to user, which are very similar to the ones preferred in the past. Collaborative Filtering (CF) recommenders recommend are those which the mentors had liked in the past. The basic assumption of recommender systems is that the mentors are people with tastes and preferences are similar to each other. Hybrid recommender systems fuses both the content and collaborative schemes while simultaneously avoiding the limitations of each of them.

The rest of the paper is structured as follows. While basics introduction is presented in section 1, Section 2 presents some background studies on recommender systems. Section 3 presents few experimental studies and finally conclusion and future work is presented in section 4.

\section{Related Work}

Collaborative filtering is one of widely used Web service recommendation technique. A key component of Web service recommendation techniques is similarity computation that using Pearson Correlation metric and several other variants. Based on this similarity computation, recommender model Personalized Hybrid Collaborative Filtering (PHCF) was developed [11]. Experiments on this real Web service were conducted using WSRec dataset which has 1.5 millions test results with 100 publicly available Web services located all around the world. A personalized instructing recommendation system (PIRS) was designed for Web-based learning task that analyzes the learning style using habits of the Web using log files. AprioriAll algorithm is used to mine the frequent patterns [12].

Collaborative Filtering $(\mathrm{CF})$ algorithm discovers similar and interested in web page targeted for specified 
users. Merge Sort Algorithm is commonly used to merge two candidate web page recommendations [13]. It is difficult to identify new items of high-quality and providing recommendations for new users. Machine learning approaches have been proposed in the past to handle the cold-start issue. In addition to the user profile, temporal information, popularity is updated [14]. Existing multimedia recommenders suggest a specific type of multimedia items rather than items of different types. This has provided a comprehensive personalized multimedia recommendation system (MudRecS) [15]. MudRecS predicts the ratings of multimedia items that match the interests of a user to make recommendations.

Ja-Hwung Su et al [16] reported on utilizing the implicit "social" factors. Integrating content-based, collaborative and information diffusion models were the key techniques involved in it. A new Personalized news Recommendation framework via implicit Social Experts (PRemiSE) was influenced on virtual social networks through implicit feedbacks was developed for recommendation process. The proposed method was able to handle cold-start problem in an efficient manner. To alleviate such problems, recommender system using FRSA (Fusion of Rough-Set and Average-category-rating) was introduced. This provides integration of multiple contents and collaborative information to predict user preferences. The proposed approach reduces the gap between the user's preferences and automated recommendations. [17]

Lack of personalized learning is one of the shortcomings of traditional e-Learning system. In order to meet out this gap, a personalized e-learning system based on intelligent agent, an intelligent agent was designed [18]. Context aware personalization system (CAPS) using ontological user profiles deemed to effective offering better recommendations to users [19]. Case-based reasoning (CBR) combined with ontology aims at providing improved recommendation system to personalize the search process and to provide users with alternate recommendations.

\section{Existing Recommender Systems}

We present some of the recommender systems existing currently. Table 1 presents different recommender systems used, domain focused \& approach/tools used. It is clearly inferred that the recommender systems focused on online web investigating on content based approaches. Subsequently fuzzy approach has been added to it. In web based experiments semantics and web server logs play a vital role [24, 25]. Fig. 1 presents a sample recommender system and the components.

Table 1. Different Recommender Systems

\begin{tabular}{lll}
\hline Recommender systems & Domain & Approach/tool used \\
\hline NewsWeeder [20] & Newsgroups & Content based \\
PRemiSE [16] & News & Social Experts \\
FTCP-RS [23] & telecom products/services & Fuzzy \\
PerHSS [24] & Hotel & Semantic web \\
YourNews [22] & Newsgroups & Content based \\
WebPUM [25] & Web & Web server logs \\
NewsDude [21] & newsgroups & Content based \\
\hline
\end{tabular}




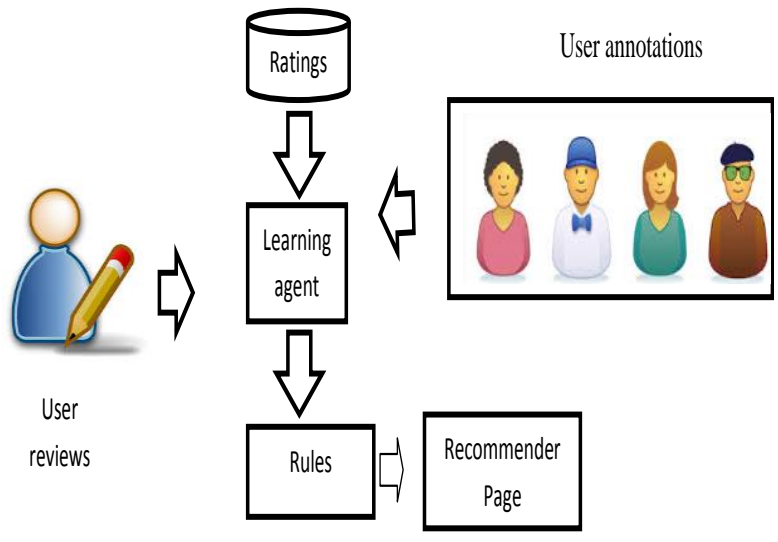

Fig.1. Sample Recommender System Architecture

The architecture shows recommender system consisting of user presenting his own reviews. These reviews would be compiled in form of ratings. The learning agent has trained set of reviews and analyses the new reviews for better classification. Rules are generated based on collective decision generated from user annotations. The final output will be recommender page.

\section{Experimental Investigations and Results}

We present a recommender system using reviews extracted from commercial websites. The reviews were available online in textual form. These were basically filtered using stop word removal and stemming. Then the reviews were analysed, similarity is calculated among each other using cosine metric or other alternate distance measures [13]. Finally the recommendation is provided to the user.

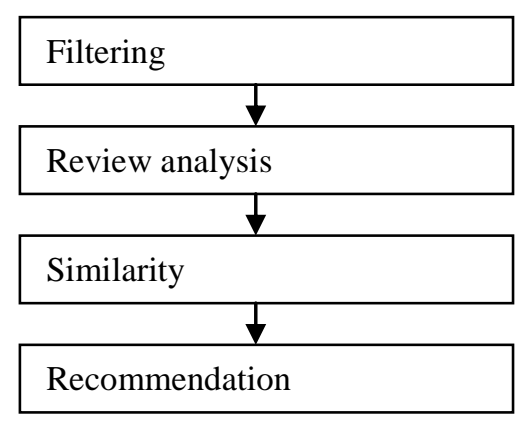

Fig.2. Steps in Recommendation Process.

Similarity is calculated using well known metrics (e.g cosine). The document is represented as a term vector by using the term frequency approach to calculate the weight of term ' $i$ ' in an article ' $\mathrm{j}$ ', as defined in Eq. ( $1 \&$ 2):

$$
W_{i, j}=f_{i, j} * \log \frac{N}{n_{i}}
$$




$$
f_{i, j}=\frac{\text { freq }_{i, j}}{\max _{l}\left(\text { freq }_{l, j}\right)}
$$

where $\mathrm{N}$ is the number of articles; $\mathrm{n}_{\mathrm{i}}$ is the number of articles that contain term $\mathrm{i} ; \mathrm{f}_{\mathrm{i}, \mathrm{j}}$ is the normalized frequency of term $i$ in article $j$; freq $q_{i, j}$ is the frequency of term $i$ in article $j$; and $\operatorname{maxl}\left(\mathrm{fl}_{\mathrm{j}}\right)$ is the frequency of term 1 which has the maximum frequency in article $\mathrm{j}$. There are various metrics which are applied in the collaborative filtering techniques to find the users' similarities.

The evaluation of the system was based on the MovieLens 10M dataset, consisting of 10 million ratings, 100,000 interactions tags applied to 10,000 users and 72,000 movies. As explicit information, we used the ratings that users assigned to items, and as implicit information. Table 2 presents a sample dataset and its charecteristics.

Table 2. Different Recommender Systems

\begin{tabular}{llll}
\hline Dataset & Rating scale (Min/max) & Total ratings & Total items \\
\hline MovieLens & $1 / 5$ & $1,000,000$ & 3500 \\
NewsDude [21] & $1 / 5$ & $2,811,983$ & 1628 \\
\hline
\end{tabular}

Precision and recall have been widely used in the field of information retrieval, to evaluate recommendation accuracy. We use the Pearson correlation coefficient as a measure of correlation between rating and predictions. These metrics have been adapted to evaluate the accuracy of a set of recommended products and are defined as:

$$
\begin{aligned}
& \text { Pr ecision }=\frac{|T \cap R|}{|R|} \\
& \text { Re call }=\frac{|T \cap R|}{|T|}
\end{aligned}
$$

where ' $\mathrm{T}$ ' is the test set and ' $\mathrm{R}$ ' is the recommended set of items for each user, respectively. An F1-metric can be used to balance the trade-off between precision and recall. These measures are calculated by considering the number of items which are either relevant or irrelevant and either recommended, which were arranged using matrix shown in Table 3. Accuracy of recommender systems is presented in Table 4.

Table 3. Confusion Matrix

\begin{tabular}{ccc}
\hline Predictor & Relevant & Irrelevant \\
\hline Recommended & $\mathrm{T}$ & $\mathrm{F}$ \\
Not recommended & $\mathrm{F}$ & $\mathrm{F}$ \\
\hline
\end{tabular}

Table 4. Confusion Matrix

\begin{tabular}{ccc}
\hline Metric & Existing & Proposed \\
\hline Precision & 0.423 & 0.513 \\
Recall & 0.293 & 0.362 \\
F1 & 0.322 & 0.401 \\
\hline
\end{tabular}




\section{Conclusion and Future Work}

The sole intention of this survey is to explore the state of art on personalized recommender systems. Recent studies performed by renowned industry groups have shown significant depth on investigating personalization, recommendations, mining and other tasks. It is evident from this paper that recommender systems have outright research platform and needs more attention with huge challenges. In particular, current recommender system for social networking environment have lots of the research attentions. We provided some helpful information to the readers who are encouraged to take up the many challenges that remain in the area. Adding multidimensional features with different modalities is our current focus which is left for future work in recommendation systems.

\section{Acknowledgements}

The authors would like to express our sincere thanks to anonymous reviewers for their insightful suggestions. The authors indeed would extend their acknowledgement for the support by all well wishers, faculty members and others who helped out in achieving this research task to get completed.

\section{References}

[1] Xi Chen, Zibin Zheng, Xudong Liu, Zicheng Huang and Hailong Sun, "Personalized QoS-Aware Web Service Recommendation and Visualization", IEEE Transactions on Services Computing, vol.6, no. 1, pp. 35-47, First Quarter 2013, doi:10.1109/TSC.2011.35.

[2] Yunhong Xu, Xitong Guo, Jinxing Hao, Jian Mac, Raymond Y.K. Lau and Wei Xu , "Combining social network and semantic concept analysis for personalized academic researcher recommendation", Decision Support Systems 54 (2012) 564-573.

[3] Qing Yang, Junli Sun, Jinqiao Wang and Zhiyong Jin, "Semantic Web-Based Personalized Recommendation System of Courses Knowledge Research", International Conference on Intelligent Computing and Cognitive Informatics (ICICCI), pp. 214 - 217, 2010 .

[4] Yu Bo and Qi Luo, "Personalized Web Information Recommendation Algorithm Based on Support Vector Machine", IEEE International Conference on Intelligent Pervasive Computing,pp. 487 - 490, 2007.

[5] Feng-Hsu Wang, "Personalized recommendation for web-based learning based on ant colony optimization with segmented-goal and meta-control strategies", IEEE International Conference on Fuzzy Systems (FUZZ), pp. 2054-2059, 2011.

[6] Ruimei Lian, "The construction of personalized Web page recommendation system in e-commerce", IEEE International Conference on Computer Science and Service System (CSSS), pp. 2687 - 2690, 2011.

[7] Xujuan Zhou, Yue Xu, Yuefeng Li and Audun Josang, "The state-of-the-art in personalized recommender systems for social networking”, Artif Intell Rev (2012) 37:119-132.

[8] Mooney RJ, Roy L (2002) Content-based book recommending using learning for text categorization. In: Proceedings of 5th ACM conference on digital libraries. ACM Press, San Antonio, pp 195-204.

[9] Resnick P, IacovouN, SuchakM, Bergstrom P, Riedl J (1994) Grouplens: an open architecture for collaborative filtering of netnews. In: CSCW. pp 175-186.

[10] Schein AI, Popescul A, Ungar LH, Pennock DM (2002) Methods and metrics for cold-start recommendations. In: SIGIR '02: Proceedings of the 25th annual international ACM SIGIR conference on research and development in information retrieval. ACM, New York, pp 253-260.

[11] Yechun Jiang, Jianxun Liu; Mingdong Tang and Xiaoqing Liu, "An Effective Web Service Recommendation Method Based on Personalized Collaborative Filtering", IEEE International Conference on Web Services (ICWS), Page(s): 211 - 218, 2011. 
[12] Liang Zhang Xiumin Liu and Xiujuan Liu, "Personalized Instructing Recommendation System Based on Web Mining", The 9th International Conference for Young Computer Scientists (ICYCS 2008), pp. 2517 $-2521,2008$.

[13] Zhongyun Ying, Zhurong Zhou; Fengjiao Han and Guofeng Zhu, "Research on personalized web page recommendation algorithm based on user context and collaborative filtering", 4th IEEE International Conference on Software Engineering and Service Science (ICSESS), Page(s): 220 - $224,2013$.

[14] Wei Chu and Seung-Taek Park, "Personalized Recommendation on Dynamic Content Using Predictive Bilinear Models", International World Wide Web Conference Committee (IW3C2), WWW 2009 MADRID, pp.69-700, 2009.

[15] Rani Qumsiyeh and Yiu-Kai Ng, "Predicting the Ratings of Multimedia Items for Making Personalized Recommendations", Proceedings of the 35th international ACM SIGIR conference on Research and development in information retrieval, pp. 475-484, 2012.

[16] Chen Lin, Runquan Xie, Xinjun Guan, Lei Li and Tao Li, "Personalized news recommendation via implicit social experts", Information Sciences, Vol. 254, pp. 1-18, 2014.

[17] Ja-Hwung Su, Bo-Wen Wang, Chin-Yuan Hsiao and Vincent S. Tseng, "Personalized rough-set-based recommendation by integrating multiple contents and collaborative information", Information Sciences 180 (2010) 113-131.

[18] Sun Duo and Zhou Cai Ying, "Personalized E- learning System Based on Intelligent Agent", 2012 International Conference on Applied Physics and Industrial Engineering, Physics Procedia (24), pp. 1899 $-1902,2012$.

[19] Ahmad Hawalah and Maria Fasli, "Utilizing contextual ontological user profiles for personalized recommendations", Expert Systems with Applications, Vo. 41, pp. 4777-4797, 2014.

[20] K. Lang, "Newsweeder: Learning to filter netnews," in Proc. of the $12^{\text {th }}$ Int. Conf. on Machine Learning, 1995, pp. 331-339.

[21] D. Billsus and M. Pazzani, "A hybrid user model for news story classification," in Proc. of the 7th Int. Conf. on User Modeling, 1999.

[22] J. Ahn, P. Brusilovsky, J. Grady, and D. He, "Open user profiles for adaptive news systems: help or harm?" in Proc. of the 16th Int. Conf. on WWW, 2007, pp. 11-20.

[23] Zui Zhang, Hua Lin, Kun Liu, Dianshuang Wu, Guangquan Zhang and Jie Lu, "A hybrid fuzzy-based personalized recommender system for telecom products/services", Information Sciences 235 (2013) 117129.

[24] Donghee Yoo, "Hybrid query processing for personalized information retrieval on the Semantic Web", Knowledge-Based Systems 27 (2012) 211-218.

[25] Mehrdad Jalali, Norwati Mustapha, Md. Nasir Sulaiman and Ali Mamat, "WebPUM: A Web-based recommendation system to predict user future movements", Expert Systems with Applications 37 (2010) 6201-6212.26.

[26] Chuanbao Wang,Fang Yuan, Ying Yun, "Tag Recommendation Based on Collaborative Filtering and Text Similarity", IJEME, vol.2, no.6, pp.7-14, 2012.

\section{Authors' Profiles}

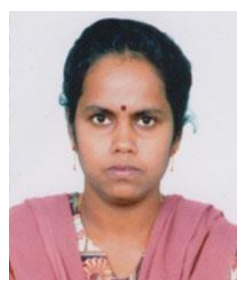

Janet Rajeswari born on $26^{\text {th }}$ June, 1976 at Tirchirappalli, Tamil Nadu, India. She completed her Undergraduate degree in Computer Science, from Bharathidasan University in 1997 and Postgraduate degree from Alagappa University, Karaikudi in 2003. She has also done M.Phil., in Computer Science in 2006 from Bharathidasan University. She is currently pursuing Ph. D., in Computer Science in Bharatiar University, Coimbatore. She is currently working as an Assistant Professor in the Department of Computer Science in Christhuraj College, Trichirappalli from $15^{\text {th }}$ June, 2012. Her areas of research interest are data mining, 
cloud computing and cryptography. She has published good number of research papers in reputed national/ international journals and conferences.

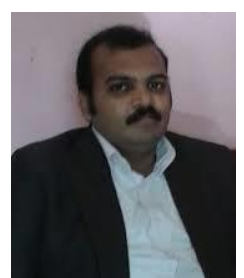

Dr.S.Hariharan received his B.E degree specialized in Computer Science and Engineering from Madurai Kammaraj University, Madurai, India in 2002, M.E degree specialized in the field of Computer Science and Engineering from Anna University, Chennai, India in 2004. He holds his Ph.D degree in the area of Information Retrieval from Anna University, Chennai, India. He is a member of various professional societies has 12 years of experience in teaching. Currently he is working as Associate Professor in Department of Computer Science and Engineering, TRP Engineering College, India. His research interests include Information Retrieval, Data mining, Opinion Mining, Web mining. He has to his credit several papers in referred journals and conferences and also serves as editorial board member and as program committee member for several international journals and conferences.

How to cite this paper: Janet Rajeswari, Shanmugasundaram Hariharan,"Personalized Search Recommender System: State of Art, Experimental Results and Investigations", International Journal of Education and Management Engineering(IJEME), Vol.6, No.3, pp.1-8, 2016.DOI: 10.5815/ijeme.2016.03.01 